\title{
Cell Speed is Independent of Force in a Mathematical Model of Amoeboidal Cell Motion with Random Switching Terms.
}

\author{
J. C. Dallon \\ dallon@math.byu.edu \\ Emily J. Evans \\ Brigham Young University \\ Christopher Grant \\ Brigham Young University \\ William V. Smith \\ Brigham Young University
}

Follow this and additional works at: https://scholarsarchive.byu.edu/facpub

Part of the Mathematics Commons

\section{Original Publication Citation}

Mathematical Bioscience 247(1) doi: 10.1016/j.mbs.2013.09.005

\section{BYU ScholarsArchive Citation}

Dallon, J. C.; Evans, Emily J.; Grant, Christopher; and Smith, William V., "Cell Speed is Independent of Force in a Mathematical Model of Amoeboidal Cell Motion with Random Switching Terms." (2013). Faculty Publications. 2718.

https://scholarsarchive.byu.edu/facpub/2718

This Peer-Reviewed Article is brought to you for free and open access by BYU ScholarsArchive. It has been accepted for inclusion in Faculty Publications by an authorized administrator of BYU ScholarsArchive. For more information, please contact ellen_amatangelo@byu.edu. 


\title{
Cell Speed is Independent of Force in a Mathematical Model of Amoeboidal Cell Motion with Random Switching Terms
}

\author{
J. C. Dallon *, E. J. Evans * , Christopher P. Grant * and W. V. Smith * \\ *Department of Mathematics, Brigham Young University, Provo, UT 84602
}

\begin{abstract}
In this paper the motion of a single cell is modeled as a nucleus and multiple integrin based adhesion sites. Numerical simulations and analysis of the model indicate that when the stochastic nature of the adhesion sites is a memoryless and force independent random process, the cell speed is independent of the force these adhesion sites exert on the cell. Furthermore, understanding the dynamics of the attachment and detachment of the adhesion sites is key to predicting cell speed. We introduce a differential equation describing the cell motion and then introduce a conjecture about the expected drift of the cell, the expected average velocity relation conjecture. Using Markov chain theory, we analyze our conjecture in the context of a related (but simpler) model of cell motion, and then numerically compare the results for the simpler model and the full differential equation model. We also heuristically describe the relationship between the simplified and full models as well as provide a discussion of the biological significance of these results.
\end{abstract}

adhesion sites | integrins | cell motion | Markov chain | switching terms

\section{Introduction}

Cell motion is fundamental in many systems including wound healing $[1,2]$, cancer [3], and morphogenesis $[4,5,6]$. In this paper we analyze the drift of an individual cell whose motion is described by an existing model which includes random switching terms [7]. The analysis reveals properties of the drift which have important implications for the measurement of cell velocities.

The main merits of the differential equation model found in [7] are the focus on discrete attachment site dynamics and the treatment of the cellular forces with the purpose of investigating whole cell migration. The modeling of discrete attachment site dynamics is not a detailed biochemical treatment; it focuses on a phenomenological treatment at the focal adhesion level. The main intent is to capture the affect of the random binding and unbinding of cell attachments on global cell motion while achieving tractable model complexity.

There are many mathematical models of cell migration $[8,9,10,11,12,13,14,15,16,17,18]$ which can be classified in diverse ways. One convenient classification involves three space-time scales: an intracellular scale, a whole cell scale, and a multi-cellular scale [19]. All of these models can be classified in one of the three categories above and each one has traits in common with the differential equation model.

The models which focus in detail on the subcellular scale include those which model different subcellular regions of the cell with viscoelastic elements like Sandersius and Newman [8]. The focus of their modeling is to capture whole cell behavior by linking subcellular forces. Other models focus on subcellular processes like actin polymerization $[9,10,11]$ or integrin attachments [12] which are essential for cell migration. Schreiber et. al. [11] investigate cell forces-velocity relationships but not at the whole cell level and their modeling is very different from ours. Paszek et. al. [12] model the in- tegrin attachment process in a detailed biochemical manner using force terms with a Hookean law to help explain integrin clustering. Although there are similar details in their modeling compared to ours, the focus is entirely different and at a different scale.

Of the four whole cell models mentioned here [13, 14, 15, 16], the first three are force based. Of the force based models two model the cytoskeletal structure and the actin polymerization forces in a phenomenological way $[13,14]$. The first uses the immersed boundary method and the second uses conservation equations, which consider the network drag, pressure, and stress to determine equations representing the flow of the network and the motion of the cell. The final force based model [15] focuses on attachments as does the model presented here. They also use Hookean laws to determine forces and cell motion but the model formulation is quite different and does not focus on the stochastic nature of the binding and unbinding events. The last whole cell model [16] is one that models motion with stochastic ordinary differential equations using white noise. It does consider the stochastic nature of the cell motion but in a very different formulation than the way it is modeled in this paper.

Finally we mention two multi-cell force based models $[17,18]$. The first uses viscoelastic forces for the cell but does not treat the binding site attachment forces in a stochastic or discrete manner [17]. The cell is treated as the fundamental object. The other model does focus on attachments in a phenomenological manner with a continuum description of integrin based adhesions [18].

The paper is organized in the following manner. In the next section we review the differential equation model for cell motion introduced in [7]. In Section 2 we also introduce the expected (average) velocity relation (EVR) conjecture by giving the equation describing the property of the expected drift of a cell. In Section 3, a related but simplified model of the cell centroid is introduced and analyzed using Markov chain theory. In Section 4 numerical results from the simplified model are compared to results from the full differential equation model. In Section 5, we give a heuristic argument that explains why the centroid model should approximate the differential equation model. Finally in Section 6, the paper concludes with a discussion of the results and their biological significance.

\section{Model and Drift}

2.1 Differential Equation Model. The cell is modeled as a nucleus and multiple interaction sites which exert force on the nucleus as shown in Figure 1. These interaction sites are integrin based adhesion sites (I-sites) [20, 21, 22]. We consider the I-sites as an adhesion complex which is part of the cell mem- 
brane and not the location of the bound complex although the distinction is not important. I-sites attach to an external substrate and once attached remain fixed to that substrate location [23]. The duration of the attachment is determined by a given probability distribution. The same is true for the time the Isite remains unattached, although the distributions need not be the same.
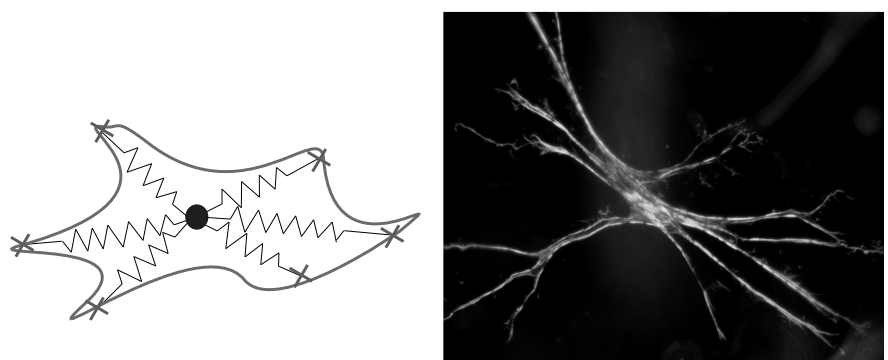

Fig. 1: The left panel depicts the way a cell is modeled mathematically. The right panel is a fibroblast in a collagen lattice. Note the similarity of the model formation with the typical spindal morphology of a fibroblast in a three dimensional lattice. The cell is a center location (nucleus) with attached springs. The other end of the springs are attached to sites which can interact with the extracellular matrix (membrane bound adhesion sites) depicted by " $x$ ".

The model assumes the I-sites exert forces on the nucleus according to Hooke's law, i.e., the force is proportional to distance. Thus it is as if the sites are attached to the cell center with springs which have a rest length assumed to be zero. Moreover, there is a drag force on the cell nucleus which is modeled assuming the center (nucleus) is a sphere in a liquid with low Reynold's number and is proportional to the velocity (Stoke's law) [17]. We denote the location of the cell center as $\mathbf{x}$, a point in $\mathbb{R}^{2}$. Likewise the location of each I-site $\mathbf{u}_{i}$ are points in $\mathbb{R}^{2}$, where $i$ ranges from 1 to $n$. Due to the low Reynolds number the acceleration term can be ignored and the equations of motion are first order [17]. The equations are

$$
C \mathbf{x}^{\prime}=\sum_{i=1}^{n}-\alpha_{i}\left(\mathbf{x}-\mathbf{u}_{i}\right) \psi_{i}(t)
$$

where $\mathbf{u}_{i}$ is given by

$$
\mathbf{u}_{i}(t)=\left\{\begin{array}{cl}
\mathbf{x}\left(t_{p_{i}}\right)+\mathbf{b}_{i} & \text { for } t_{p_{i}} \leq t<t_{p_{i}}+w_{p_{i}} \text { and } \psi_{i} \neq 0 \\
0 & \text { otherwise }
\end{array},\right.
$$

$t_{p_{i}}$ is the most recent time that $\psi_{i}$ made the transition from 0 to 1 , and $w_{p_{i}}$ is the duration time that $\psi_{i}$ remains 1 for the most recent transition. Here, $\psi_{i}$ is the random variable which indicates if the $i$ th I-site is attached. The spring constants which define the cell force are $\alpha_{i}$, and the drag coefficient is $C$. Finally $\mathbf{b}_{i}$ are random vectors that describe the outreach to the I-sites from the nucleus.

2.2 EVR Conjecture. Numerical simulations given in [7] indicate that the cell speed for the differential equation model appears to be independent of the spring constants $\alpha_{i}$ (see Figure 2) for a wide range of physiologically relevant values. These numerical simulations led to the following conjecture about the cell drift which is referred to as the EVR (expected average velocity relation) conjecture. In the next section we prove an EVR result for a simplified model under special conditions.

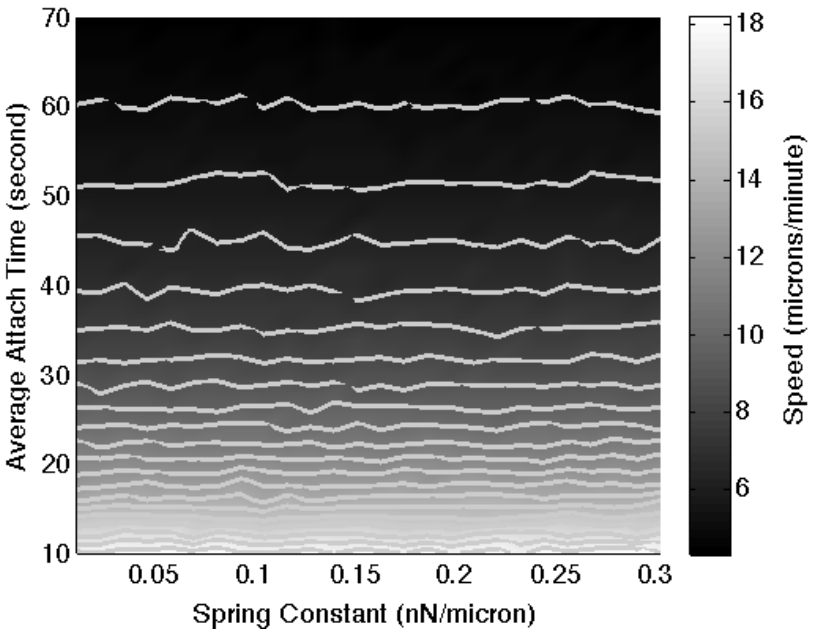

Fig. 2: The speed of a single cell is plotted in grayscale against the mean attach time and strength of the cell. The cell speed is remarkably constant with respect to the strength of the cell. The contour lines are plotted over the shading. The plot shows the average of 50 random runs for each data point. The mean detach time is 25 seconds with a Poisson distrirandom runs for each data point. The mean detach time is 25 seconds with a Poisson distri-
bution and the attach time is taken from a Poisson distribution. The speed was calculated in the simulations by averaging over a 60 second time interval.

The EVR for the differential equation model may be stated very simply as

$$
\lim _{\zeta \rightarrow \infty} E[\mathbf{x}(t+\Delta t)-\mathbf{x}(t)]=\lambda
$$

where $\zeta=\alpha C^{-1}$ and $\alpha=\min \alpha_{i}$. Here $\Delta t$ is an arbitrary positive increment of time bounded away from zero. The meaning of the EVR is that the expectation of the increment of motion approaches a constant value as the ratio of the spring constants to the drag increases. Recall that $\mathbf{x}(t)$ is the location of the cell and is a random process and therefore the expectation is taken relative to the outcome space. For further details see [24] and for a concrete example see Section 3.2.

To better understand this conjecture we first consider a simplified model where the differential equation becomes an algebraic equation. We will assume that the cell instantly moves to the equilibrium position, i.e., moves instantly to its centroid. In this simplified model elapsed time is replaced by an index which depends on the latest binding event.

\section{Simplified Centroid Model}

3.1 The Model. In this section we consider a simpler but related problem in order to better understand the EVR conjecture for the differential equation model. The differential equation model can be approximated heuristically by a problem that tracks the centroid of the cell, $\mathbf{c}^{j}$. This new problem is motivated by informally considering the limit of our model as the cell spring constants, $\alpha_{i}$ in Equation (1), become very large. In this limit, one expects the cell nucleus to jump from centroid to centroid. Let $j$ denote the number of binding events (attach or detach events) that have occurred and $n$ the number of I-sites. The equation describing $\mathbf{c}^{j}$ is

$$
0=\sum_{i=1}^{n} \alpha_{i}\left(\mathbf{c}^{j}-\mathbf{v}_{i}^{j}\right) \psi_{i}^{j}
$$


where $\mathbf{v}_{i}^{j}$ is the location of the $i$ th attachment site at stage $j$.

3.2 Analysis of the centroid model. We begin by describing a formal setting for the centroid model. Let $n \in \mathbb{N}, r>0$, and a probability measure $\eta$ on the Borel sets of $\mathbb{R}^{2}$ be given. Consider a cell in 2-dimensional space with $n$ I-sites, each of which has a position and a status, either "attached" or "detached". (Since the numerical calculations are for cells in $\mathbb{R}^{2}$ we restrict ourselves to two dimensions, but all the results are valid for a cell in $\mathbb{R}^{N}$.) The state of the cell evolves through a sequence of stages in the following way. At each stage, exactly one of the I-sites randomly changes status, with the probability of a given detached I-site changing status being $r$ times the probability of a given attached I-site changing status. If the I-site's status goes from detached to attached in stage $j$ then its location changes to $\mathbf{b}_{i}^{j}+\mathbf{c}$, where $\mathbf{b}_{i}^{j}: \Omega \rightarrow \mathbb{R}^{2}$ is an $\eta$-distributed random variable independent of $\left\{\mathbf{b}_{i}^{k}: k<j, 1 \leq i \leq n\right\}$, and $\mathbf{c}$ is the centroid of the locations of I-sites that were attached in stage $j-1$ unless there were no such I-sites; if no I-sites were attached in stage $j-1, \mathrm{c}$ is the location of the last I-site to detach. (If the I-site detaches, it does not move.)

Letting $\psi_{i}$ be 1 if I-site $i$ is attached and 0 if it's detached, and letting $\mathbf{v}_{i}$ be the location of the $i$ th I-site, the state of the organism is conveniently viewed as an element in

$$
\begin{aligned}
X:= & \left\{\left(\psi_{1}, \ldots, \psi_{n}\right),\left(\mathbf{v}_{1}, \ldots, \mathbf{v}_{n}\right), \mathbf{c}\right) \in\{0,1\}^{n} \times\left(\mathbb{R}^{2}\right)^{n} \times \mathbb{R}^{2} \\
& \left.: \sum_{i=1}^{n} \psi_{i}\left(\mathbf{v}_{i}-\mathbf{c}\right)=0\right\} .
\end{aligned}
$$

(Endow $X$ with the product topology, where $\{0,1\}$ has the discrete topology and $\mathbb{R}$ has its standard topology.) The ccomponent of the state represents the value of $\mathbf{c}$ that will be used in the next stage, if it is an attachment stage. Given subsets $A_{1}, A_{2}, \ldots, A_{n}$ of $\{0,1\}$, Borel subsets $B_{1}, B_{2}, \ldots, B_{n}$ of $\mathbb{R}^{2}$, and a Borel subset $D$ of $\mathbb{R}^{2}$, we can compute the probability that an organism in state $s^{j}:=\left(\left(\psi_{1}, \ldots, \psi_{n}\right),\left(\mathbf{v}_{1}, \ldots, \mathbf{v}_{n}\right), \mathbf{c}\right)$ at stage $j$ will have a state $s^{j+1}$ contained in $S:=\left(A_{1} \times \cdots \times A_{n}\right) \times$ $\left(B_{1} \times \cdots \times B_{n}\right) \times D$ at stage $j+1$ as follows.

1. There are $n$ different I-sites that can change status. Each attached I-site has a certain probability $p$ of changing status, and each detached I-site has probability $r p$ of changing status. There are $|\psi|:=\sum_{i=1}^{n} \psi_{i}$ of the former and $(n-|\psi|)$ of the latter. Since exactly one I-site changes status, we know that $|\psi| p+(n-|\psi|) r p=1$, so $p=1 /(|\psi|+(n-|\psi|) r)$ and $r p=r /(|\psi|+(n-|\psi|) r)$.

2. Given that the $i$ th I-site changes status, $s^{j+1}$ will certainly not be in $S$ unless $1-\psi_{i} \in A_{i}$ and $\psi_{k} \in A_{k}$ for all $k \neq i$. Since I-sites only move when they change status (from detached to attached), $s^{j+1}$ will also not be in $S$ unless $\mathbf{v}_{k} \in B_{k}$ for all $k \neq i$.

3. Given that the $i$ th I-site changes status, we consider 3 cases:

(a) Suppose $|\psi|=\psi_{i}=1$. Then the $i$ th I-site will detach and not move, and (since this will result in all I-sites detached) the final component of $s^{j+1}$ will equal $\mathbf{v}_{i}$, which equals c, since $s^{j} \in \mathrm{X}$. Thus, $s^{j+1}$ will not be in $S$ unless $\mathbf{v}_{i} \in B_{i}$ and $\mathbf{c} \in D$.

(b) Suppose $|\psi|>\psi_{i}=1$. Then the $i$ th I-site will detach and not move, while the centroid of attached I-sites will move to adjust for the $i$ th I-site's absence. Thus, $\sum_{k=1}^{n} \psi_{k}\left(\mathbf{v}_{k}-\mathbf{c}\right)=0$ and $\sum_{k \neq i} \psi_{k}\left(\mathbf{v}_{k}-\hat{\mathbf{c}}\right)=0$, where $\hat{\mathbf{c}}$ is the last component of $s^{j+1}$. Solving these two equations for $\hat{\mathbf{c}}$ yields $\hat{\mathbf{c}}=\mathbf{c}+\left(\mathbf{c}-\mathbf{v}_{i}\right) /(|\psi|-1)$, and $s^{j+1}$ will not be in $S$ unless $\mathbf{v}_{i} \in B_{i}$ and $\hat{\mathbf{c}} \in D$.

(c) Suppose $\psi_{i}=0$. Then the $i$ th I-site will attach and move to a new location $\hat{\mathbf{v}}_{i}$ whose displacement from $\mathbf{c}$ is given by $\eta$, and the location of the centroid will change from c to some new value $\hat{\mathbf{c}}$, specified by the two equations $\sum_{k=1}^{n} \psi_{k}\left(\mathbf{v}_{k}-\mathbf{c}\right)=0$ and $\sum_{k=1}^{n} \psi_{k}\left(\mathbf{v}_{k}-\hat{\mathbf{c}}\right)+\hat{\mathbf{v}}_{i}-\hat{\mathbf{c}}=0$. These two equations imply that $\hat{\mathbf{v}}_{i}$ will be in $B_{i}$, and $\hat{\mathbf{c}}$ will be in $D$ if and only if $\hat{\mathbf{v}}_{i}-\mathbf{c}$ is in $\left(B_{i}-\mathbf{c}\right) \cap(|\psi|+1)(D-\mathbf{c})$. To see this, note that $\hat{\mathbf{v}}_{i}-\hat{\mathbf{c}}=-\sum_{k=1}^{n} \psi_{k}\left(\mathbf{v}_{k}-\hat{\mathbf{c}}\right)=-\sum_{k=1}^{n} \psi_{k}(\mathbf{c}-\hat{\mathbf{c}})$ thus $\hat{\mathbf{v}}_{i}+|\psi| \mathbf{c}=(|\psi|+1) \hat{\mathbf{c}}$ and $\hat{\mathbf{v}}_{i}-\mathbf{c}=(|\psi|+1)(\hat{\mathbf{c}}-\mathbf{c})$.

Thus, the probability of transitioning from state $s^{j}$ to $S$ is

$$
\begin{gathered}
\left.\mathcal{P}\left(\left(\psi_{1}, \ldots, \psi_{n}\right),\left(\mathbf{v}_{1}, \ldots, \mathbf{v}_{n}\right), \mathbf{c}\right),\left(\chi_{i=1}^{n} A_{i}\right) \times\left(\chi_{i=1}^{n} B_{i}\right) \times D\right):= \\
\sum_{i=1}^{n} \frac{r+(1-r) \psi_{i}}{r n+(1-r)|\psi|} \delta_{1-\psi_{i}}\left(A_{i}\right)\left(\prod_{k \neq i} \delta_{\psi_{k}}\left(A_{k}\right) \delta_{\mathbf{v}_{k}}\left(B_{k}\right)\right) \times \\
\begin{cases}\eta\left(\left(B_{i}-\mathbf{c}\right) \cap(|\psi|+1)(D-\mathbf{c})\right) & \text { if } \psi_{i}=0, \\
\delta_{\mathbf{v}_{i}}\left(B_{i}\right) \delta_{\mathbf{c}}(D) & \text { if }|\psi|=\psi_{i}=1, \\
\delta_{\mathbf{v}_{i}}\left(B_{i}\right) \delta_{\mathbf{c}+\left(\mathbf{c}-\mathbf{v}_{i}\right) /(|\psi|-1)}(D) & \text { if }|\psi|>\psi_{i}=1 .\end{cases}
\end{gathered}
$$

This formula extends to a transition probability kernel $\mathcal{P}$ : $\mathrm{X} \times \mathcal{B}(\mathrm{X}) \rightarrow[0,1]$ (where $\mathcal{B}(\cdot)$ denotes the collection of Borel sets of a topological space). It can be shown that for any initial distribution $\rho$ on $\mathcal{B}(\mathrm{X})$, there is a probability measure $\mathbb{P}_{\rho}$ on $\mathcal{B}\left(X^{\mathbb{N}}\right)$ and a stochastic process $\Phi$ such that

$$
\begin{array}{r}
\mathbb{P}_{\rho}\left(\Phi_{0} \in A_{0}, \Phi_{1} \in A_{1}, \ldots, \Phi_{k} \in A_{k}\right)= \\
\int_{y_{k-1} \in A_{k-1}} \cdots \int_{y_{0} \in A_{0}} \rho\left(d y_{0}\right) \mathcal{P}\left(y_{0}, d y_{1}\right) \cdots \mathcal{P}\left(y_{k-1}, A_{k}\right) ;
\end{array}
$$

i.e., $\boldsymbol{\Phi}$ is a Markov chain on $\left(\mathrm{X}^{\mathbb{N}}, \mathcal{B}\left(\mathrm{X}^{\mathbb{N}}\right), \mathbb{P}_{\rho}\right)$ with initial distribution $\rho$ [25]. (Here, we follow the convention that if $\lambda$ is a measure on a set $A$, then $\int_{x \in A} \lambda(d x):=\int_{A} d \lambda$.)

Because $\eta$-distributed random vectors $\mathbf{b}_{i}^{j}$ describing the outreach to the I-sites from the centroid need not be rotationally symmetric, it is not expected that there is a stationary distribution associated with the transition kernel $\mathcal{P}$. There is, however, a simple Markov chain connected with $\mathcal{P}$ that has a stationary distribution. This is the embedded Markov chain associated with the attachment-detachment status of each Isite.

Consider the stochastic process governing the evolution of $|\psi|$ alone; it has a state space of $\{0, \ldots, n\}$ and a transition matrix $\left(P_{i \ell}\right)_{i, \ell=0}^{n}$ given by the formula

$$
P_{i \ell}= \begin{cases}i /((n-i) r+i) & \text { if } \ell-i=-1 \\ (n-i) r /((n-i) r+i) & \text { if } \ell-i=1 \\ 0 & \text { otherwise }\end{cases}
$$

Thus $P_{i \ell}$ is the probability that the state transitions from $i$ Isites bound to $\ell$ I-sites bound. Since for any state $i$ there is a positive probability for a transition either up $i+1$ or down $i-1$ at every stage, the Markov chain is irreducible. The state space is finite so the Markov chain is positive recurrent and there exists a unique stationary distribution. That is, there is a vector $\left(\pi_{0}, \ldots, \pi_{n}\right)$ with non-negative components such that $\sum_{i} \pi_{i}=1$ and $\sum_{i} \pi_{i} P_{i \ell}=\pi_{\ell}$ [26]. The stationary distribution $\left(\pi_{0}, \ldots, \pi_{n}\right)$ 
of this latter chain is given by the formulas $\pi_{0}=1 /\left(2(1+r)^{n-1}\right)$ and

$$
\pi_{k}=\frac{r^{k-1}(1-r)\left(\begin{array}{c}
n-1 \\
k-1
\end{array}\right)+r^{k}\left(\begin{array}{l}
n \\
k
\end{array}\right)}{2(1+r)^{n-1}}
$$

for $0<k \leq n$.

We now propose the following theorem.

Theorem 1. Let $\mathbb{E}_{\rho}$ be the expectation operator corresponding to $\mathbb{P}_{\rho}$, and let $\mathbb{E}[\eta]=\int_{\mathbb{R}^{2}} x d \eta$, the expected value of any $\eta$-distributed random variable; then

$$
\begin{aligned}
& \mathbb{E}_{\rho}\left[\mathbf{c}^{j}-\mathbf{c}^{j-1}\right]= \\
& \left(1+\sum_{k=1}^{n} \frac{\left(r^{k-1}(1-r)\left(\begin{array}{l}
n-1 \\
k-1
\end{array}\right)+r^{k}\left(\begin{array}{l}
n \\
k
\end{array}\right)\right) r(n-k)}{(k+r(n-k))(k+1)}\right) \frac{\mathbb{E}[\eta]}{2(1+r)^{n-1}} .
\end{aligned}
$$

Proof. Let $\rho$ be any probability measure on $\mathcal{B}(\mathrm{X})$ such that

$$
\rho\left(\left(\left\{\left(\psi_{1}, \ldots, \psi_{n}\right)\right\} \times\left(\mathbb{R}^{2}\right)^{n} \times \mathbb{R}^{2} \cap \mathrm{X}\right)=\pi_{|\psi|}\right.
$$

for every $\left(\psi_{1}, \ldots, \psi_{n}\right) \in\{0,1\}^{n}$, and consider the evolution of a Markov chain $\boldsymbol{\Phi}=\Phi_{0}, \Phi_{1}, \ldots$ on $\left(X^{\mathbb{N}}, \mathcal{B}\left(X^{\mathbb{N}}\right), \mathbb{P}_{\rho}\right)$ with initial distribution $\rho$. Let $\Phi_{j}=\left(\left(\psi_{1}^{j}, \ldots, \psi_{n}^{j}\right),\left(\mathbf{x}_{1}^{j}, \ldots, \mathbf{x}_{n}^{j}\right), \mathbf{c}^{j}\right)$ and $\left|\psi^{j}\right|:=\sum_{i=1}^{n} \psi_{i}^{j}=k$ for each $j$.

If $|\psi|=k$, then there is probability $k /(k+r(n-k))$ that the next change will involve detachment, and probability $r(n-$ $k) /(k+r(n-k))$ that the next change will involve attachment. Also, $\mathbb{E}_{\rho}\left[\mathbf{c}^{j+1}-\mathbf{c}^{j}|| \psi^{j+1}|=k-1,| \psi^{j} \mid=k\right]=0$ (by symmetry), while $\mathbb{E}_{\rho}\left[\mathbf{c}^{j+1}-\mathbf{c}^{j}|| \psi^{j+1}|=k+1,| \psi^{j} \mid=k\right]=\mathbb{E}[\eta] /(k+1)$. Using these conditional expected values and the formula for $\pi_{k}$, we see that the expected translation

$$
\begin{aligned}
& \mathbb{E}_{\rho}\left[\mathbf{c}^{j}-\mathbf{c}^{j-1}\right]=\sum_{k=0}^{n} \pi_{k} \cdot \frac{r(n-k)}{k+r(n-k)} \cdot \frac{\mathbb{E}[\eta]}{k+1} \\
& =\left(1+\sum_{k=1}^{n} \frac{\left(r^{k-1}(1-r)\left(\begin{array}{c}
n-1 \\
k-1
\end{array}\right)+r^{k}\left(\begin{array}{c}
n \\
k
\end{array}\right)\right) r(n-k)}{(k+r(n-k))(k+1)}\right) \times \\
& \frac{\mathbb{E}[\eta]}{2(1+r)^{n-1}} .
\end{aligned}
$$

For the special case when $r=1$ this formula simplifies to

$$
\mathbb{E}_{\rho}\left[\mathbf{c}^{j}-\mathbf{c}^{j-1}\right]=\mathbb{E}[\eta] \frac{\left(2^{n}-1\right)}{n 2^{n}} .
$$

As the number of I-sites becomes very large the right hand side of equation 5 asymptotically approaches the function

$$
\mathbb{E}[\eta] \frac{(r+1)}{2 r n} .
$$

\section{Numerical Verification}

In this section we compare numerical results for both the centroid model and the differential equation model with the theoretical results. To numerically solve the centroid model we use a random number generator to generate $\mathbf{b}_{i}^{j}$, set $\mathbf{v}_{i}^{j}=\mathbf{c}^{j-1}+\mathbf{b}_{i}^{j}$, and then solve Equation (3). The vectors $\mathbf{b}_{i}^{j}$ were generated by choosing an angle from a uniform distribution in the interval $-\pi$ to $\pi$ and choosing a length from a uniform distribution on the interval 0 to 10 . We then determine the cell drift by averaging the cell displacement over the number of binding events. To numerically solve the differential equation model, we again generate the vectors $\mathbf{b}_{i}^{j}$ in the same manner but we must also generate attach times and detach times. Then starting at time $t$ and continuing to time $t+\Delta t$, we solve equation 1 using the software package CVODE [27], update the binding status as necessary at the I-sites, and repeat the process with new initial conditions for the differential equation and the new location for the adhesion site. In this numerical solution all the binding events that happen in the time interval $\Delta t$ occur simultaneously at the end of the interval. The cell drift is determined in the same manner as for the centroid model.

We numerically verify the expected value given by [5], by simulating the centroid model and calculating the average drift of multiple runs. Figure 3 shows the results of this comparison where ' $x$ ' denotes the results from the simulations and the solid line is calculated from [5]. Anticipating the results should be similar for the differential equation model, we compare simulations for Equation (1) to the theoretical prediction where all the spring constants are the same, thus $\alpha_{i}=\alpha$ for all $i$, and where the waiting time for both attachment and detachment of I-sites are exponentially distributed with a mean attach time of 20 and a mean detach time of 60 , making the random component a Markov process. We hypothesize that for larger values of $\frac{\alpha}{C}$ the theoretical result will better predict the expected drift. The numerical results confirm our hypothesis and are shown in Figure 4 for three values of the ratio $\frac{\alpha}{C}$. Physiologically realistic ratios of $\frac{\alpha}{C}$ are between $24.9-900$ minute $^{-1}$. These are determined by considering that cell forces range from $0.116-4.44 \mathrm{nN}$ per micron [7] and assuming $C$ is the drag coefficient of a sphere of 1.5 micron radius in water (about $0.28 \mathrm{nN}$ s per micron). In Figure 5, simulations with different distributions for the waiting times are compared.

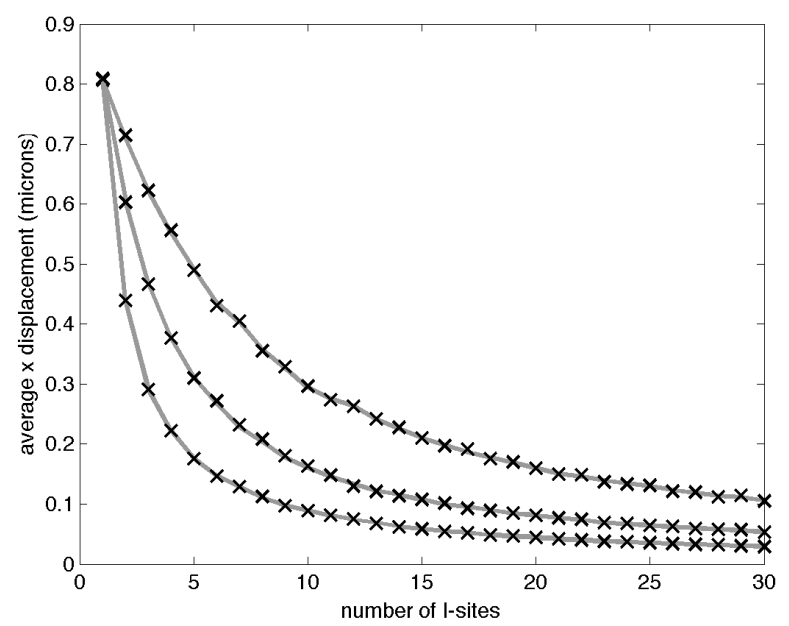

Fig. 3: This figure compares the numerical simulations for the centroid model (3) with the theoretical results (5). The ' $x$ ' denote the numerical simulations and the line shows the theoretical results. The horizontal axis indicates the number of I-sites for the cell and the vertical axis shows the average displacement per binding event in the $x$ direction (results are similar for the $y$ direction). The three different set of ' $x$ ' and lines in decreasing order are for $r=1 / 3$, $r=1$, and $r=10$. Other values of $r$ show equally good fits. The displacement is in microns. The simulations had 100,000 binding events for each run with a fixed number of I-sites.

The mean attach time for all the distributions is 20 and the mean detach time for all the distributions is 60 . When 
the distributions are exponential the process has the Markov property and the fit is good. Simulations with Poisson distributions or normal distributions give expected drifts which are different from the theoretical prediction indicating the results do not hold for processes which have a memory.

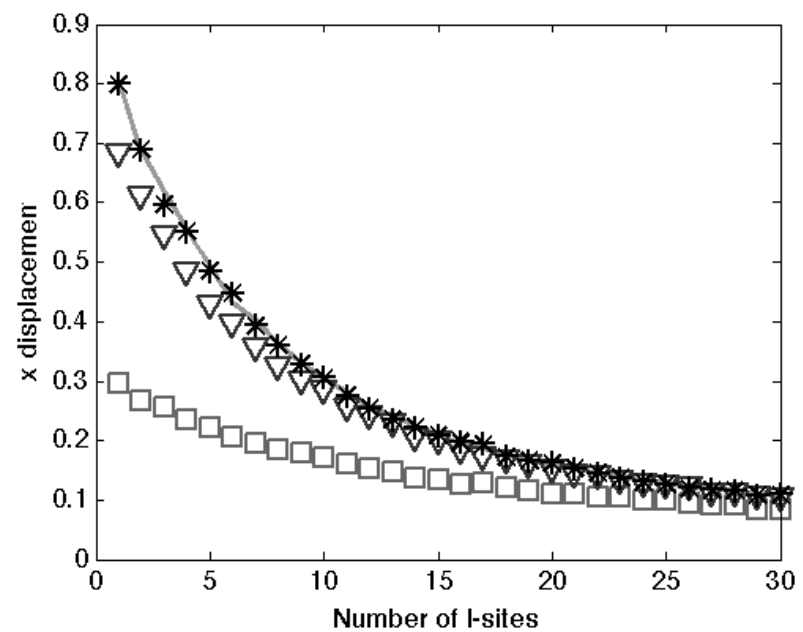

Fig. 4: This figure compares the numerical simulations for the differential equation model (1) with the theoretical result (5). The symbols indicate the numerical simulations and the line shows the theoretical results. The squares denote results with the ratio of $\frac{\alpha}{C}=16.6$, the triangles a ratio of 166.6 , and the * a ratio of 1666.6. The horizontal axis indicates the number of I-sites for the cell and the vertical axis shows the average displacement per binding event in the $x$ direction. The simulations had 100,000 binding events for each run with a fixed num ber of I-sites. The distribution for the attach time was an exponential distribution with mean 20 and the distribution for the detach time was exponential with mean 60. The time interval $\Delta t=1$.

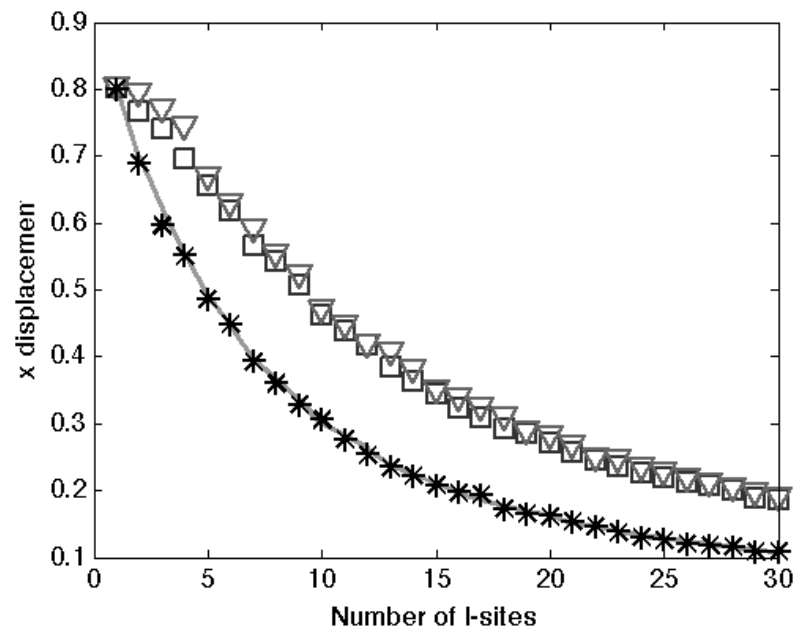

Fig. 5: This figure shows a comparison between the numerical simulations for the differential equation model (1) with different waiting time distributions and the theoretical results (5). The symbols indicate the numerical simulations and the line shows the theoretical results. The denote simulations with exponential distributions, the triangle normal distributions with viation 1 , and the square Poisson distributions. The ratio of $\frac{\alpha}{C}=1666.6$. The horizontal axis indicates the number of I-sites for the cell and the vertical axis shows the average displacement per binding event in the $x$ direction. The simulations had 100,000 binding events for each run with a fixed number of I-sites. Distributions for the attach time had mean 20 and the distributions for the detach time had mean 60 for all the simulations. The time interval $\Delta t=1$.
5 Heuristic Relation Between the Centroid and Differential Equation Models

As stated previously for most physiologically realistic parameters we expect the centroid model to approximate the differential equation model. Consider the differential equation model (Equation (1)) with initial condition $\mathbf{x}^{o}, \mathbf{u}_{i}^{o}$, and $\psi_{i}^{o}$ for each $i$. For $t<\tau$ ( $\tau$ corresponds to the first adhesion site binding event), Equation (1) has the solution

$$
\mathbf{x}(t)=\frac{\sum_{i} \alpha_{i} \mathbf{u}_{i} \psi_{i}}{\sum_{i} \alpha_{i} \psi_{i}}+\left(\mathbf{x}^{o}-\frac{\sum_{i} \alpha_{i} \mathbf{u}_{i} \psi_{i}}{\sum_{i} \alpha_{i} \psi_{i}}\right) e^{-\frac{\sum_{i} \alpha_{i} \psi_{i} t}{C}} .
$$

The solution changes as $t$ passes the first binding time, but its form remains similar. It is reasonable to expect that $\mathbf{x}(t)$ will approach the behavior of

$$
\frac{\sum_{i} \alpha_{i} \mathbf{u}_{i} \psi_{i}}{\sum_{i} \alpha_{i} \psi_{i}}
$$

provided event times do not crowd together. If they do not crowd together the exponential term in the solution is dominated by the behavior of $t$ and hence tends to zero. It is also reasonable to assume that the term

$$
\mathbf{x}^{o}-\frac{\sum_{i} \alpha_{i} \mathbf{u}_{i} \psi_{i}}{\sum_{i} \alpha_{i} \psi_{i}}
$$

is bounded and therefore dominated by the exponential.

The centroid model,

$$
0=\sum_{i=1}^{n} \alpha_{i}\left(\mathbf{c}-\mathbf{v}_{i}\right) \psi_{i}
$$

has the solution

$$
\frac{\sum_{i} \alpha_{i} \mathbf{v}_{i} \psi_{i}}{\sum_{i} \alpha_{i} \psi_{i}}
$$

and therefore may be thought of as a limit system for the differential equation model.

\section{Discussion}

In this paper we analyzed a mathematical model of cell motion which was previously introduced in [7]. Numerical results there indicated that measured cell speed was independent of the force the cell exerted. These results inspired the EVR (expected average velocity relationship) conjecture presented here. The first step to understand the EVR conjecture was to analyze a simplified centroid model. A formula for the expected drift of cell motion governed by this model was determined using Markov chain theory. A heuristic argument for why the centroid model should approximate the differential equation model was given. Numerical results indicate that the EVR conjecture is accurate and that the centroid model does approximate the differential equation model for a wide range of physiologically relevant values. Additional numerical results considered the differential equation model with different distributions for the binding dynamics. Future work will explore the behavior of the cells for more biologically relevant binding dynamic distributions, i.e. not memoryless and force dependent.

Experimental data has focused recently on measuring cell forces and speeds $[28,29,30,31,32,33,34,35,36,30,37,38$, $39,40]$ and not on the statistical behavior of adhesion sites. In Figure 6 we show data from simulations discussed in [7]. In those simulations the differential equation model (1) is used to calculate average cell speed for a range of cell strengths and a range of mean attach times. The mean detach time is fixed and the cell forces for each I-site are set to be the same, i.e. $\alpha=\alpha_{i}$ for all $i$. As the figure shows for a range of values the correct 
cell behavior (in terms of speed and force) can be predicted by the model. The range of values for $\alpha$ and for the mean attach time respectively are $0.232-4.44 \mathrm{nN} /$ micron and $810-1210 \mathrm{sec}-$ onds for fibroblasts, $1.17-2.33 \mathrm{nN} /$ micron and $28-88$ seconds for neutrophils, $0.233-0.614 \mathrm{nN} /$ micron and $230-408$ seconds for murine dendritic cells, $1.17-1.18 \mathrm{nN} /$ micron and 206-408 for endothelial cells, and $0.116-0.348 \mathrm{nN} /$ micron and $18-70$ seconds for Dd cells. Figure 7 shows the same data plotted using the mean attach time as the vertical axis. Comparing the two figures one can see that there is no functional relationship between the cell force and the cell speed whereas there appears to be a simple power relationship between the attach time of the I-sites and the cell speed (the figure is a log log plot).

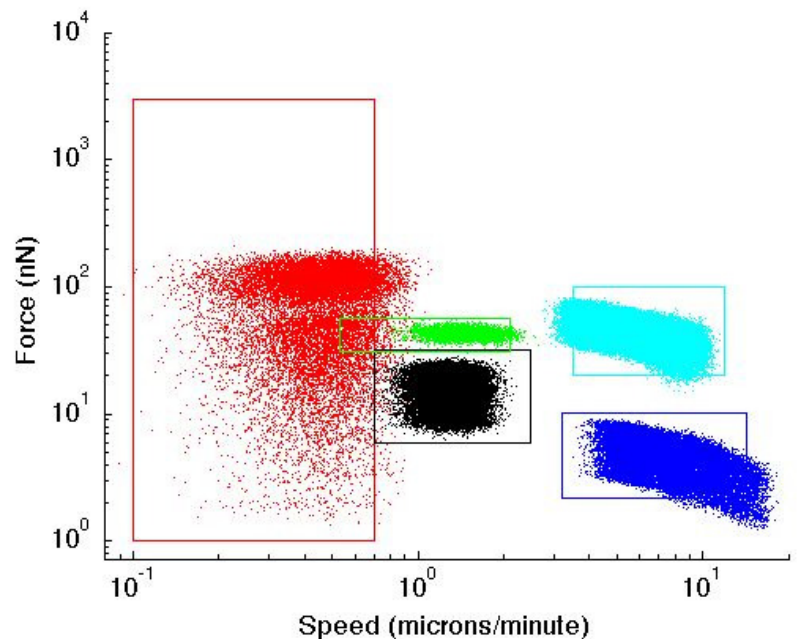

Fig. 6: The boxes roughly outline the regions where experimental data has been reported for different cells types. The scatter plots are simulation results with parameters used to mimic the behavior of the different cells using the differential equation model. Red denotes fibroblasts, black denotes murine dendritic cells, cyan denotes neutrophils, green denotes endothelial cells, and blue denotes Dd cells. Figure taken from [7] with permission.

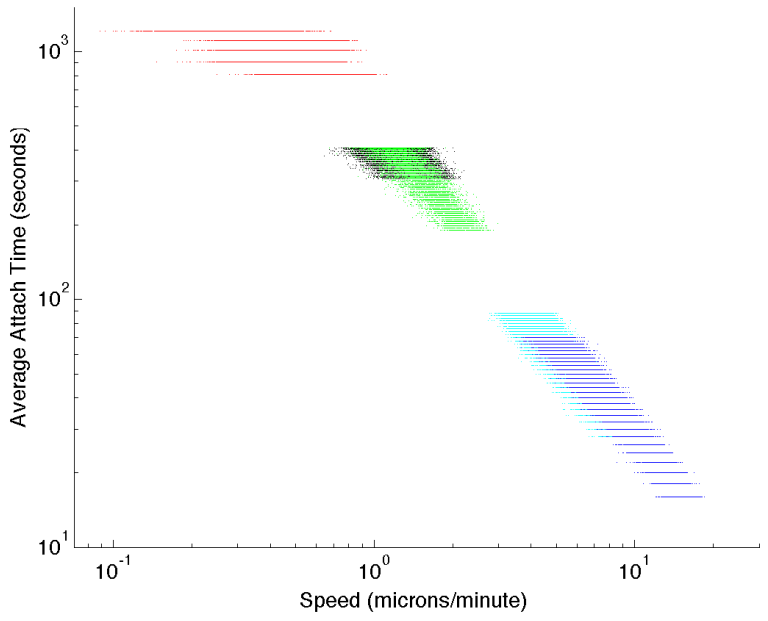

Fig. 7: The same data plotted in Figure 6 is plotted with the vertical axis as the mean attach time. Notice that there appears to be a linear relationship between the logarithms of the two variables. Yet in the previous figure there is no functional relationship between the force and the speed. Red denotes fibroblasts, black denotes murine dendritic cells, cyan denotes neutrophils, green denotes endothelial cells, and blue denotes Dd cells.

For experimentalists this work indicates that measuring cell force is not enough to determine the cell speed and likewise measuring cell speed is not enough to determine cell force. The reason for this is cell speed is typically measured by averaging cell displacement on the order of minutes. However, the saltatory motion of cells occurs on a shorter time scale and this saltatory motion is more closely related to the exerted force. New adhesions sites cause the cell to shift quickly after the adhesion site is formed as do detachment events at the adhesion site. Between these binding events the cell remains relatively stationary, yet substrate tension can remain high. Our conjecture says that in the limit force is not important in determining cell speed when measured by averaging displacement over larger time scales. The model predicts that averages of cell displacement on the order of minutes (cell speed) will depend on the binding dynamics of the adhesion sites in particular the average attach time. 
1. Krawczyk, W. (1971) A pattern of epidermal cell migration during wound healing. The Journal of cell biology 49:247-263.

2. Tanner, K., Ferris, D., Lanzano, L., Mandefro, B., Mantulin, W., Gardiner, D., Rugg E., and Gratton, E. (2009) Coherent movement of cell layers during wound healing by image correlation spectroscopy. Biophysical journal 97:2098-2106.

3. Yilmaz, M. and Christofori, G. (2010) Mechanisms of motility in metastasizing cells. Molecular Cancer Research 8:629-642.

4. Keller, R., Davidson, L., Edlund, A., Elul, T., Ezin, M., Shook, D., and Skoglund, P. (2000) Mechanisms of convergence and extension by cell intercalation. Philosophical Transactions of the Royal Society of London. Series B: Biological Sciences 355:897922.

5. Mammoto, T. and Ingber, D. (2010) Mechanical control of tissue and organ development. Development 137:1407-1420.

6. Rieu, J.-P., Saito, T., Delanoë-Ayari, H., Sawada, Y., and Kay, R. R. (2009) Migration of dictyostelium slugs: anterior-like cells may provide the motive force for the prespore zone. Cell Motil Cytoskeleton 66:1073-86.

7. Dallon, J. C., Scott, M., and Smith, W. V. (2013) A force based model of individua cell migration with discrete attachment sites and random switching terms. Journal of Biomechanical Engineering 135:071008-071008-10.

8. Sandersius, S. A. and Newman, T. J. (2008) Modeling cell rheology with the subcellular element model. Physical Biology 5:015002.

9. Keren, K., Pincus, Z., Allen, G. M., Barnhart, E. L., Marriott, G., Mogilner, A. and Theriot, J. A. (2008) Mechanism of shape determination in motile cells. Nature 453:475-U1.

10. Mogilner, A. and Oster, G. (2003) Force generation by actin polymerization ii: The elastic ratchet and tethered filaments. Biophysical Journal 84:1591-1605.

11. Schreiber, C. H., Stewart, M., and Duke, T. (2010) Simulation of cell motility that reproduces the force-velocity relationship. Proceedings of the National Academy of Sciences of the United States of America 107:9141-9146.

12. Paszek, M. J., Boettiger, D., Weaver, V. M., and Hammer, D. A. (2009) Integrin clustering is driven by mechanical resistance from the glycocalyx and the substrate. Plos Computational Biology 5:e1000604.

13. Bottino, D. and Fauci, L. (1998) A computational model of ameboid deformation and locomotion. European Biophysics Journal With Biophysics Letters 27:532-539.

14. Herant, M. and Dembo, M. (2010) Form and function in cell motility: From fibroblasts to keratocytes. Biophysical Journal 98:1408-1417.

15. Buenemann, M., Levine, H., Rappel, W.-J., and Sander, L. M. (2010) The role of cell contraction and adhesion in dictyostelium motility. Biophysical Journal 99:50-58.

16. Ionides, E., Fang, K., Isseroff, R., and Oster, G. (2004) Stochastic models for cell motion and taxis. Journal of Mathematical Biology 48:23-37.

17. Dallon, J. C. and Othmer, H. G. (2004) How cellular movement determines the collective force generated by the Dictyostelium discoideum slug. J. Theor. Biol. 231:203222.

18. Mallet, D. G. and Pettet, G. J. (2006) A mathematical model of integrin-mediated haptotactic cell migration. Bulletin of Mathematical Biology 68:231-253.

19. Dickinson, R. (2000) A generalized transport model for biased cell migration in an anisotropic environment. Journal of Mathematical Biology 40:97-135.

20. Friedl, P. and Gilmour, D. (2009) Collective cell migration in morphogenesis, regeneration and cancer. Nature Reviews Molecular Cell Biology 10:445-457.
21. Ulrich, F. and Heisenberg, C.-P. (2009) Trafficking and cell migration. Traffic 10:811-

22. Gumbiner, B. M. (1996) Cell adhesion: the molecular basis of tissue architecture and morphogenesis. Cell 84:345-57.

23. Lauffenburger, D. and Horwitz, A. (1996) Cell migration: A physically integrated molecular process. Cell 84:359-369.

24. Klenke, A. (2007) Probability theory: a comprehensive course. Springer.

25. Meyn, S. P., Tweedie, R. L., and Glynn, P. W. (2009) Markov chains and stochastic stability. Cambridge University Press Cambridge.

26. Meini, B. (2007) Markov chains. In Hogben, L., Brualdi, R., Greenbaum, A., and Mathia, R. (eds.), Handbook of Linear Algebra. Chapman \& Hall, CRC, New York.

27. Woodward, C., Hindmarsh, A. C., and Serban, R. (2012) SUNDIALS (Suite of Nonlinear and DIfferential/ALgebraic equation Solvers). Copyright $\odot 2002$, The Regents of the University of California. Produced at the Lawrence Livermore National Laboratory.

28. Fray, T. R., Molloy, J. E., Armitage, M. P., and Sparrow, J. C. (1998) Quantification of single human dermal fibroblast contraction. Tissue Eng 4:281-91.

29. Wrobel, L. K., Fray, T. R., Molloy, J. E., Adams, J. J., Armitage, M. P., and Sparrow J. C. (2002) Contractility of single human dermal myofibroblasts and fibroblasts. Cel Motility and the Cytoskeleton 52:82-90.

30. Tymchenko, N., Wallentin, J., Petronis, S., Bjursten, L. M., Kasemo, B., and Gold J. (2007) A novel cell force sensor for quantification of traction during cell spreading and contact guidance. Biophysical Journal 93:335-345.

31. Galbraith, C. G. and Sheetz, M. P. (1997) A micromachined device provides a new bend on fibroblast traction forces. Proc Natl Acad Sci U S A 94:9114-8.

32. Munevar, S., Wang, Y., and Dembo, M. (2001) Traction force microscopy of migrating normal and h-ras transformed 3t3 fibroblasts. Biophysical Journal 80:1744-1757.

33. Brown, R., Prajapati, R., McGrouther, D., Yannas, I., and Eastwood, M. (1998) Tensional homeostasis in dermal fibroblasts: Mechanical responses to mechanical loading in three-dimensional substrates. Journal of cellular physiology 175:323-332.

34. Delvoye, P., Wiliquet, P., Levêque, J. L., Nusgens, B. V., and Lapière, C. M. (1991) Measurement of mechanical forces generated by skin fibroblasts embedded in a threedimensional collagen gel. J Invest Dermatol 97:898-902.

35. Kaiser, J.-P., Reinmann, A., and Bruinink, A. (2006) The effect of topographic characteristics on cell migration velocity. Biomaterials 27:5230-5241.

36. Nenasheva, T. A., Carter, T., and Mashanov, G. I. (2012) Automatic tracking of individual migrating cells using low-magnification dark-field microscopy. Journal of Microscopy 246:83-88.

37. Ricart, B. G., Yang, M. T., Hunter, C. A., Chen, C. S., and Hammer, D. A. (2011) Measuring traction forces of motile dendritic cells on micropost arrays. Biophysical Journal 101:2620-2628.

38. Jannatt, R. A., Dembo, M., and Hammer, D. A. (2011) Traction forces of neutrophils migrating on compliant substrates. Biophysical Journal 101:575-584.

39. Smith, L. A., Aranda-Espinoza, H., Haun, J. B., Dembo, M., and Hammer, D. A (2007) Neutrophil traction stresses are concentrated in the uropod during migration. Biophysical Journal 92:L58-L60.

40. Delanoë-Ayari, H., Iwaya, S., Maeda, Y. T., Inose, J., Rivière, C., Sano, M., and Rieu, J.-P. (2008) Changes in the magnitude and distribution of forces at different dictyostelium developmental stages. Cell Motil Cytoskeleton 65:314-31. 